DOI: $10.3901 / J M E .2018 .01 .090$

\title{
基于视觉辅助的隧道轮廓监测"
}

\author{
李华 雷勇 甘创 \\ (浙江大学流体动力与机电系统国家重点实验室 杭州 310027)
}

\begin{abstract}
摘要: 隧道掘进机(Tunnel boring machine, TBM)被广泛地运用于隧道施工中。隧道的变形是影响 TBM 施工安全的主要因素之 一, 过大的隧道变形会引起隧道塌方、TBM 卡机等事故, 影响施工进度, 威胁施工人员的生命安全。针对 TBM 施工情况, 介绍基于视觉辅助的隧道轮廓监测系统。采用荧光涂料设计人工标志点, 单目视觉检测目标, 云台驱动激光位移传感器瞄准 目标。基于极坐标测量原理, 获取目标点三维坐标。为进一步提高测量效率, 提出基于半定规划的双目视觉系统辅助测量方 案。试验结果表明系统的精度可达 $1 \mathrm{~mm}$ 并具有较高的测量效率。
\end{abstract}

关键词: TBM; 隧道变形; 视觉; 激光

中图分类号: TG156

\section{Tunnel Deformation Monitoring Based on Vision Assistant}

\author{
LI Hua LEI Yong GAN Chuang
}

(State Key Laboratory of Fluid Power and Mechatronic Systems, Zhejiang University, Hangzhou 310027)

\begin{abstract}
Tunnel boring machine (TBM) has been widely applied in tunnel construction. The deformation of tunnel is one of the major concerns in TBM construction project. Major deformation may cause tunnel landslide or TBM jam, influence the construction schedule and threaten the safety of the constructors. A tunnel deformation monitoring system based on vision assistant in TBM construction is proposed. Artificial target points are designed by using fluorescent coating. Targets are detected by using monocular vision and aimed by using a Pan-tilt platform. The three-dimensional coordinates of the targets are obtained based on the principal of polar coordinates of the Pan-tilt platform using laser distance measurement. The efficiency of measuring is further improved by using a binocular vision system assistant measurement based on semi-definite programming method. Experiments show the precision of the system can be up to $1 \mathrm{~mm}$ with good efficiency.
\end{abstract}

Key words: TBM; tunnel deformation; vision; laser

\section{0 前言}

全断面硬岩掘进机(Tunnel boring machine, $\mathrm{TBM})$, 是高度机械化和自动化的大型隧道开挖祄砌 成套设备, 在相同条件下, 掘进速度大约为传统钻 爆法的 3 10 倍, 当隧道长度与直径的比值大于 600 时, 采用 $\mathrm{TBM}$ 进行隧道施工是经济的 ${ }^{[1]}$ 。我国对 $\mathrm{TBM}$ 的需求越来越大, 然而 TBM 设备庞大, 对地 质条件的适应性不如钻爆法灵活 ${ }^{[2]}$ 。隧道变形过大 可能会引起 TBM 卡机, 导致长时间停机, 严重的 会导致支护失效、隧道塌方造成施工人员及设备的 损失。因此需要对隧道变形进行不断的监测, 才能

* 国家重点基础研究发展计划(973 计划, 2013CB035405)和国家自然科学 基金创新研究群体科学基金(51521064)资助项目。20170302 收到初稿, 20170523 收到修改稿
保证隧道施工的安全进行。

有关隧道变形监测方法, 国内外展开了许多研 究。其主要可以分为两类: 接触式测量和非接触式 测量。

传统的接触式测量方法有：收敛计、倾斜仪、 水准仪等方法, 这些测量方法精度较高, 然而自动 化程度不高, 耗费较多人力和物力且无法得到测量 点完整的位置信息。巴塞特收敛系统 ${ }^{[3]}$ 通过在一个 隧道断面内将含有倾角传感器的单元交接成环, 可 以获得测量点在断面内的二维坐标，但是无法测量 纵向的变形且安装不便。布里沸光时域反射计 ${ }^{[4]}$ 采 用光纤介质中的布里渊光时域反射原理测量隧道表 面的应变，易实现长距离、全方位检测，且光纤材 料防水和防腐, 但是其空间分辨率只能达到 $1 \mathrm{~m}$, 且光纤较脆弱, 在施工过程中容易造成损伤。

传统的非接触式测量方法有：全站仪、三维激 
光扫描仪、和摄影测量等方法。全站仪由光电测距 仪、电子经纬仪和数据处理系统等组成, 对目标点 进行逐点测量。三维激光扫描仪可获取隧道表面的 三维点云数据。摄影测量法运用数字图像处理技术 获取目标点的三维坐标。但是现有的非接触式测量 方法不适合用于 TBM 施工情况, 由于 TBM 在向前 移动, 需要对测量装置重复标定, 且测量结果容易 受到振动、粉尘等环境因素的影响。因此需要隧道 变形监测系统跟随 TBM 移动并对开挖初期的隧道 表面进行自动化监测。

本文根据 TBM 的施工情况, 提出了基于视觉辅 助的隧道变形监测系统, 并设计了人工标志点施工方 案和试验装置, 建立了试验装置的数学模型, 利用图 像处理提取目标中心像素坐标以及基于图像的视觉 伺服控制瞄准目标, 通过激光测量部分点的三维坐 标。同时, 提出了双目视觉测量目标点相对距离, 利 用半定规划求得其余目标点三维坐标的方法以提高 测量效率, 并通过仿真分析了各参数对测量精度的影 响。搭建试验平台验证了测量系统的精度和效率。

\section{1 变形监测方案}

将测量装置固定于如图 1 所示的位置, 跟随 TBM 向前移动, 由于 TBM 的开挖直径大约为 $8 \mathrm{~m}$, 则测量系统的测量范围为 $2 \sim 5 \mathrm{~m}$ 。根据铁路隧道监 控量测技术规程, 测量系统的精度应在 $1 \mathrm{~mm}$ 以下。

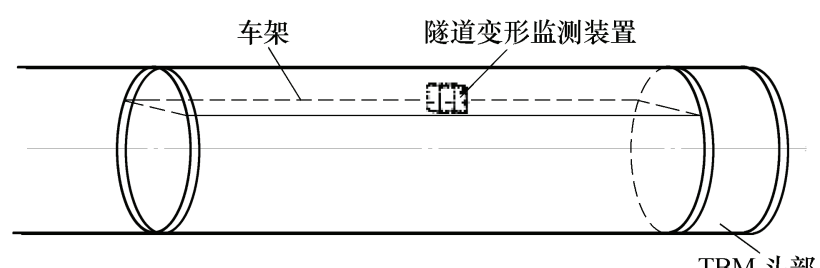

图 1 测量装置的安装示意图

如图 2 所示, 在隧道围岩表面或者针杆上涂覆 苂光涂料作为人工标志点, 通过紫外光照射, 荧光 涂料在光线较暗的情况下也容易识别。该方法易于 操作, 且荧光涂料不易受振动、粉尘等的影响。

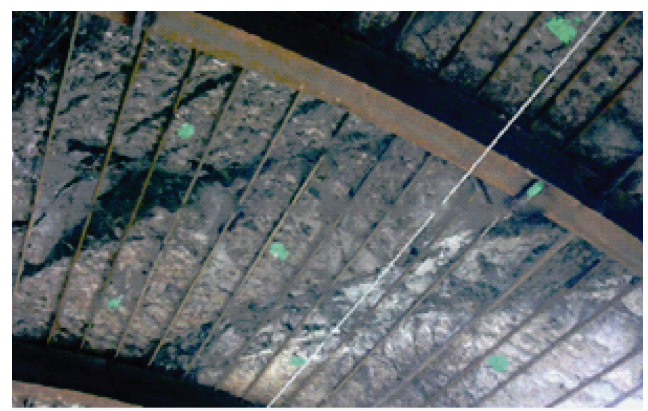

图 2 隧道环境
本文设计了如图 3 所示的试验装置。其主要由 单目相机、激光位移传感器、云台、分光镜、遮光 板及双目相机组成, 单目相机用于识别和瞄准目标, 激光位移传感器获取测量装置与目标点之间的距离 信息。云台带动相机及激光位移传感器等获得相应 的转角信息。分光镜采用窄带滤光片, 其能通过大 部分的激光, 而反射其他可见光。遮光板采用黑色 植线布材料, 可以吸收激光, 并遮挡环境光。

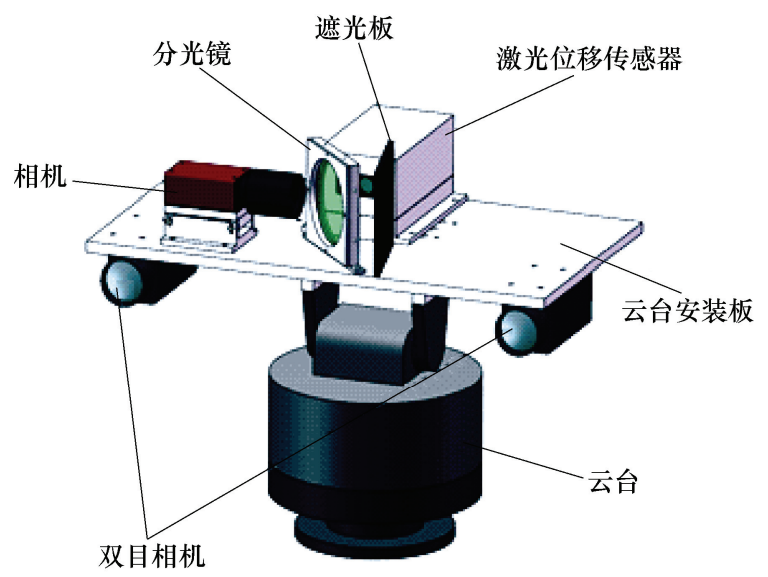

图 3 测量装置的三维模型

隧道变形监测系统的工作流程如图 4 所示。利 用测量装置在隧道内进行扫描, 单目相机寻找到一 些目标点, 并通过一定的方式使得激光束对其中的 目标点进行逐一的瞄准和测量, 再利用双目视觉测 量部分目标点之间的相对距离, 得到其余目标点的 三维坐标。若在两次测量之间 TBM 发生了移动, 对移动前后的三维坐标进行拼接。

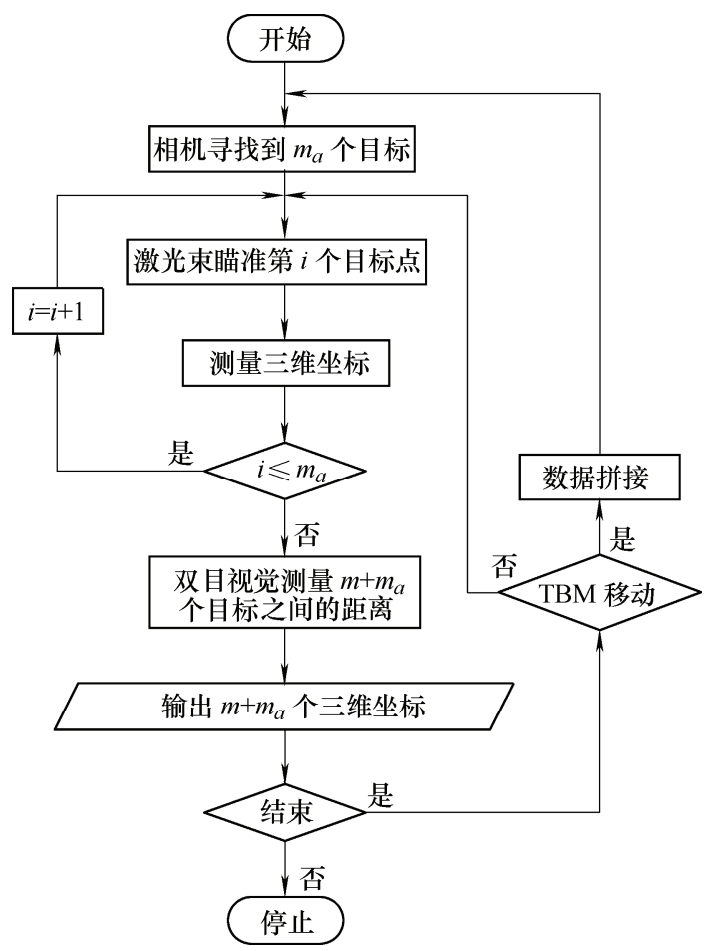

图 4 隧道变形监测系统的工作流程 


\section{2 测量装置的数学模型}

建立测量装置的 D-H 模型如图 5 所示, 其中 $Z_{0}$ 轴与云台的水平旋转轴重合, $Z_{1}$ 轴与云台的坚直俯 仰轴重合, $Z_{c}$ 与相机等效光轴重合。其关节参数表 如表 1 所示，为了方便计算 $D_{1}$ 取为 0 。

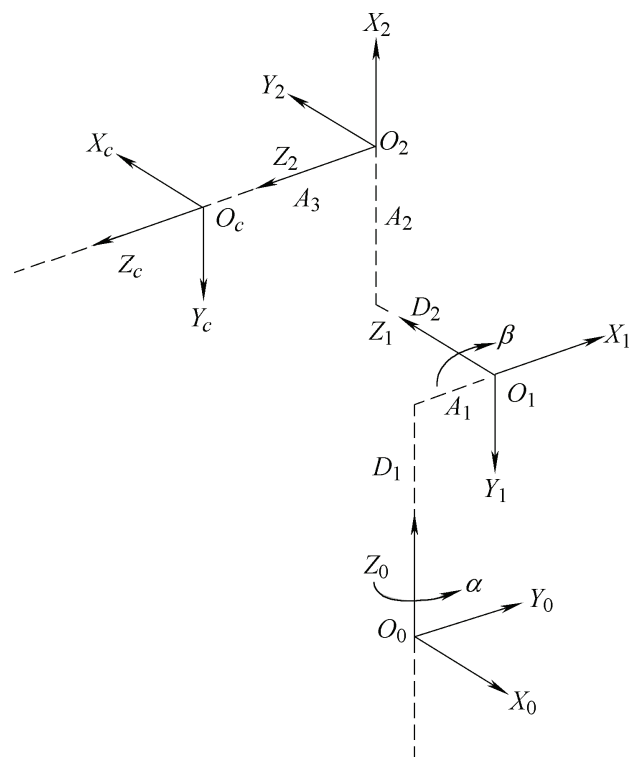

图 5 D-H 模型描述

表 1 关节参数表

\begin{tabular}{ccccc}
\hline & 扭角 $\alpha_{i} / \mathrm{rad}$ & 杆长 $a_{i} / \mathrm{mm}$ & 转角 $\theta_{i} / \mathrm{rad}$ & 杆间距 $d_{i} / \mathrm{mm}$ \\
\hline 1 & $-\pi / 2$ & $A_{1}$ & $\alpha$ & $D_{1}$ \\
2 & $\pi / 2$ & $A_{2}$ & $\beta$ & $D_{2}$ \\
$c$ & 0 & $A_{3}$ & $\pi / 2$ & 0 \\
\hline
\end{tabular}

依据 D-H 坐标变换, 将表 1 中的参数代入相 邻坐标变换矩阵中, 得到坐标 0 到坐标系 2 的变 换矩阵

$$
\begin{gathered}
{ }_{2}^{0} \boldsymbol{T}={ }_{1}^{0} \boldsymbol{T}_{2}^{1} \boldsymbol{T}=\left(\begin{array}{cccc}
\mathrm{c} \alpha \mathrm{c} \beta & -\mathrm{s} \alpha & \mathrm{c} \alpha \mathrm{s} \beta & t_{2 x} \\
\mathrm{~s} \alpha \mathrm{c} \beta & \mathrm{c} \alpha & \mathrm{s} \alpha \mathrm{s} \beta & t_{2 y} \\
-\mathrm{s} \beta & 0 & \mathrm{c} \beta & t_{2 z} \\
0 & 0 & 0 & 1
\end{array}\right) \\
t_{c x}=A_{1} \mathrm{c} \alpha+A_{2} \mathrm{c} \alpha \mathrm{c} \beta-D_{2} \mathrm{~s} \alpha \\
t_{c y}=A_{1} \mathrm{~s} \alpha+A_{2} \mathrm{~s} \alpha \mathrm{c} \beta+D_{2} \mathrm{c} \alpha \\
t_{c z}=D_{1}-A_{2} \mathrm{~s} \beta
\end{gathered}
$$

坐标 0 到坐标系 $c$ 的变换矩阵为

$$
\begin{gathered}
{ }_{c}^{0} \boldsymbol{T}={ }_{2}^{0} \boldsymbol{T}_{c}^{2} \boldsymbol{T}=\left(\begin{array}{cccc}
-\mathrm{s} \alpha & -\mathrm{c} \alpha \mathrm{c} \beta & \mathrm{c} \alpha \mathrm{s} \beta & t_{c x} \\
\mathrm{c} \alpha & -\mathrm{s} \alpha \mathrm{c} \beta & \mathrm{s} \alpha \mathrm{s} \beta & t_{c y} \\
0 & \mathrm{~s} \alpha & \mathrm{c} \beta & t_{c z} \\
0 & 0 & 0 & 1
\end{array}\right) \\
t_{c x}=A_{1} \mathrm{c} \alpha+A_{2} \mathrm{c} \alpha \mathrm{c} \beta-A_{3} \mathrm{~s} \alpha-D_{2} \mathrm{~s} \alpha
\end{gathered}
$$

$$
t_{c y}=A_{1} \mathrm{~s} \alpha+A_{2} \mathrm{~s} \alpha \mathrm{c} \beta+A_{3} \mathrm{~s} \alpha+D_{2} \mathrm{c} \alpha
$$

$$
t_{c z}=D_{1}-A_{2} \mathrm{~s} \beta
$$

如果目标点在 $Z_{2}$ 轴上, 由激光位移传感器的 测量可得其距 $O_{2}$ 的距离 $k$, 再根据云台返回的角 度信息 $\alpha$ 和 $\beta$, 可得到目标点在基坐标系 0 的坐 标为

$$
\left(\begin{array}{c}
X_{0} \\
Y_{0} \\
Z_{0} \\
1
\end{array}\right)={ }_{2}^{0} \boldsymbol{T}(\alpha, \beta)\left(\begin{array}{l}
0 \\
0 \\
k \\
1
\end{array}\right)
$$

由于目标点距离相机较远 $(2 \sim 5 \mathrm{~m})$, 因此相机 的光学成像模型可以近似为针孔模型, 则摄像机 坐标系与图像像素坐标系之间的坐标变换为 ${ }^{[5]}$

$$
Z_{c}\left(\begin{array}{l}
u \\
v \\
1
\end{array}\right)=\left(\begin{array}{cccc}
\alpha_{x} & 0 & u_{0} & 0 \\
0 & \alpha_{y} & v_{0} & 0 \\
0 & 0 & 1 & 0
\end{array}\right)\left(\begin{array}{c}
X_{c} \\
Y_{c} \\
Z_{c} \\
1
\end{array}\right)
$$

式中, $(u, v)$ 表示图像像素坐标系下的坐标, $\alpha_{x}, \alpha_{y}$ 分别表示焦距 $f$ 在 $u$ 和 $v$ 方向的像素。 $\left(u_{0}, v_{0}\right)$ 表示光 轴与图像平面的焦点, 即图像主点。通过张正友标 定法 ${ }^{[6]}$ 可对相机内参进行标定。

\section{3 目标点的提取与瞄准}

\section{1 原始图像的颜色矫正}

经窄带滤光片反射的图像如图 6 所示。图像 呈现出渐变的蓝绿色, 原因是窄带滤光片的滤光 特性随入射角的变化发生了改变。如图 7 所示, 等效相机在窄带滤光片的入射光路与相机的光路 相同。入射角从左到右逐渐变大，窄带滤光镜透 射曲线朝着短波长方向移动 ${ }^{[7]}$ ，导致透过的红光 逐渐增加, 而反射的红光逐渐减小，从而呈现蓝 绿色。

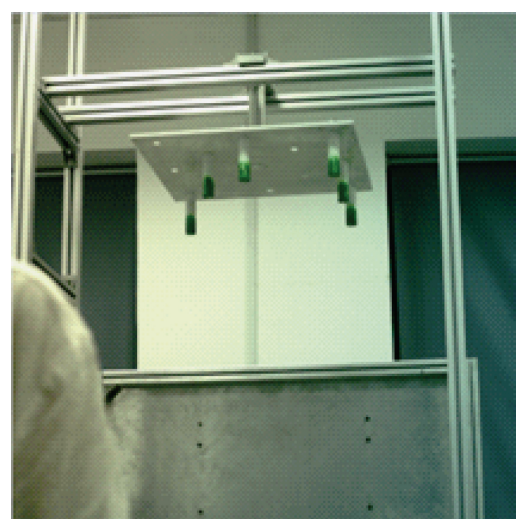

图 6 原始图像 


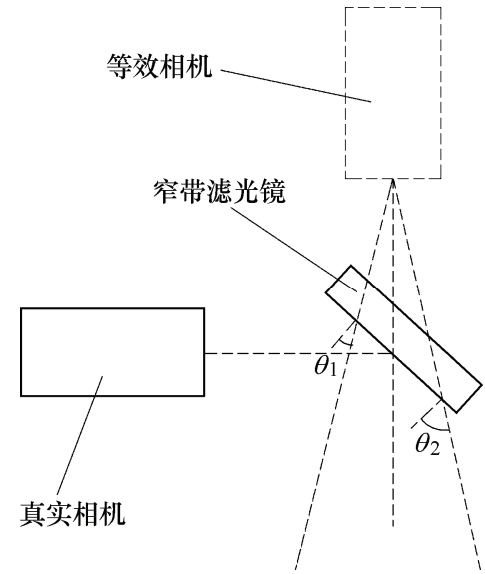

图 7 试验装置光路示意图

图像颜色矫正的方法是对减少的红光进行补 偿。其算法如下所示

$$
\begin{aligned}
& \text { for } i=1: W \\
& \qquad R(:, i)=R(:, i) *(1+\varepsilon * i / W) \\
& \text { end }
\end{aligned}
$$

式中, $W$ 表示图像的像素宽度; $R$ 表示图像某点红 色通道的灰度值; $\varepsilon$ 表示修正因子。则可得到矫正 后的图像如图 8 所示。

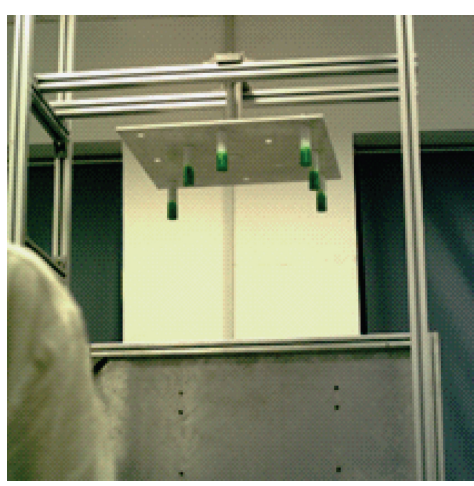

图 8 颜色矫正后的图像

\section{2 目标点中心提取}

为了测量目标点, 首先需要获取目标点的中心 在相机图像中的坐标。

提出了基于 $\mathrm{RGB}$ 模型的阈值分割法以将目 标点与背景区分, 首先将三维 RGB 模型转化成二 维模型: Value $1=G-R$, Value $2=G-B$ 。遍历图 像中的每个像素, 若 $\operatorname{Valuel}(i, j)>$ Thresh1 且 Value2 $(i, j)>$ Thresh2, 则该点的灰度值置为 255 , 否则置为 0 , 其中 Thresh 1 、Thresh 2 为选取的阈值, 可通过最大类间方差法 ${ }^{[8]}$ 得到。由于阈值分割后的 图像存在杂点, 因此利用形态学运算中的腐蚀和 膨胀进行图像处理。阈值分割前后的图像如图 9 所示。

利用 Canny 算子 ${ }^{[8]}$ 进行边缘检测, 提取出目标 点的边缘。

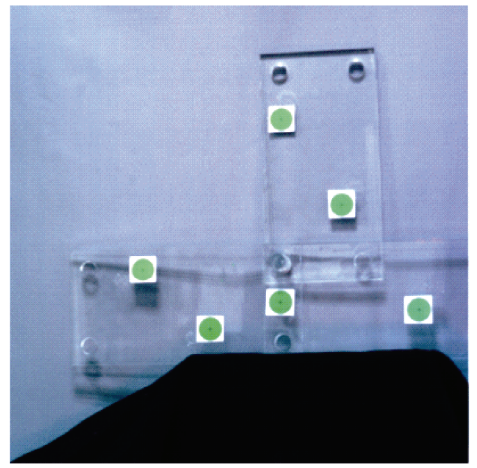

(a) 阈值分割处理前

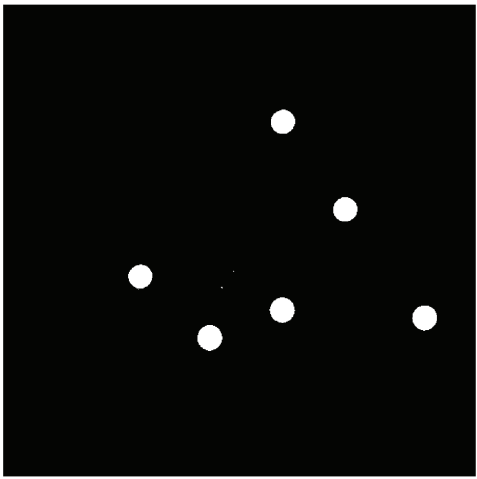

(b) 國值分割处理后

图 9 阈值分割前后的图像

利用图像矩计算得到目标中心。若图像的零阶 矩 $M_{00}=\sum_{i} \sum_{j} V(i, j), u$ 方向的一阶矩 $M_{10}=\sum_{i} \sum_{j} i V(i, j), M_{01}=\sum_{i} \sum_{j} j V(i, j)$, 则目标中 心的坐标为: $i_{c}=M_{10} / M_{00}, j_{c}=M_{01} / M_{00}$, 其中 $V(i, j)$ 表示像素点的灰度值。其结果如图 10 所示。

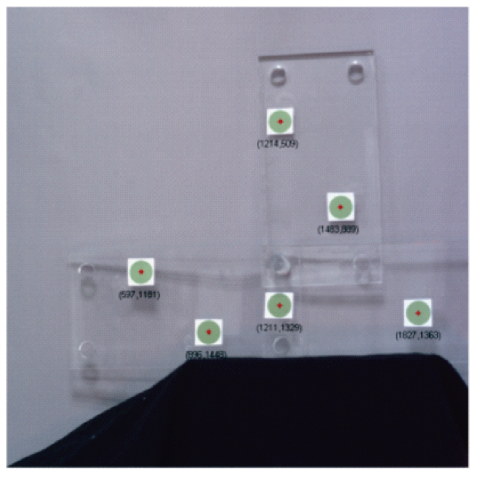

图 10 形心提取结果

\section{3 目标点的瞄准}

根据单目相机捕获的图像，需要控制云台使得 激光束瞄准目标点，此时目标点在图像中的坐标与 图像主点重合。

目前视觉伺服控制主要包括基于图像的视觉伺 服、基于位置的视觉伺服以及混合的视觉伺服控制。 
基于位置的视觉伺服控制需要图像空间到位置 空间的完整精确映射, 而测量装置采用单目相机进 行瞄准, 无法得到完整映射, 因此采用基于图像的 视觉伺服控制。目前基于图像的视觉伺服控制大多 基于图像的雅可比矩阵 ${ }^{[9]}$, 但是需要对深度进行实 时估计。PID 控制不需要建立模型, 然而需要对目 标进行不断的跟踪, 由于隧道内目标点的模式相似, 容易造成跟踪错误。目标点相对于基坐标系几乎静 止不动, 于是提出了一种迭代控制用于目标的瞄准, 其流程图如 11 所示, 其中 $T$ 为图像中瞄准误差國值, $\Delta_{\min }$ 为云台的步距角。 $k_{\alpha} 、 k_{\beta}$ 分别为云台 $\alpha$ 角和 $\beta$ 角的比例系数, 其值可由如下公式近似得到

$$
\left\{\begin{array}{l}
\Delta a \approx \Delta u / \Delta u_{\max } \arctan \left(\Delta u_{\max } / l\right)=k_{a} \Delta u \\
\Delta b \approx \Delta v / \Delta v_{\text {max }} \arctan \left(\Delta v_{\text {max }} / l\right)=k_{\beta} \Delta u
\end{array}\right.
$$

式中, $l$ 为焦距 $f$ 的像素尺寸, 因此

$$
\begin{aligned}
& k_{\alpha}=\arctan \left(\Delta u / \Delta u_{\text {max }}\right) / \Delta u_{\text {max }} \\
& k_{\beta}=\arctan \left(\Delta v / \Delta v_{\text {max }}\right) / \Delta v_{\text {max }}
\end{aligned}
$$

为了使控制最终收玫, 每次迭代时 $k_{\alpha}$ 和 $k_{\beta}$ 都需要更 新, 累乘一个小于 1 的正整数。其控制结构图如图 12 所示, 其中 $u_{0} 、 v_{0}$ 为图像主点坐标, $u 、 v$ 为目标 点形心坐标, $\alpha 、 \beta$ 为云台需要转过的角度。

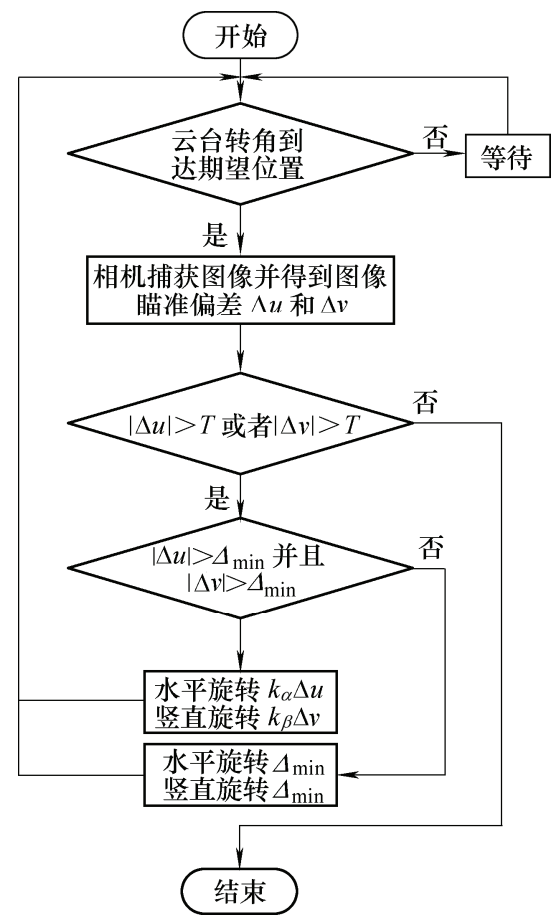

图 11 控制流程图

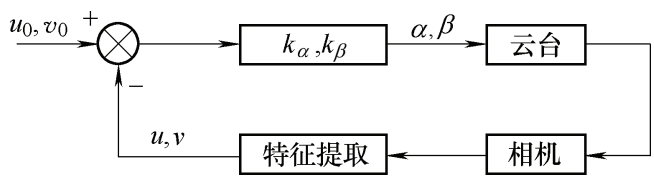

图 12 控制结构图

\section{4 三维坐标的求取与拼接}

\section{1 优化问题}

基于单目视觉和激光测距的方式需要逐点对目 标点进行测量, 测量速度较慢。为了进一步提高测 量效率, 提出利用激光测量部分点, 双目视觉测量 部分点对间的相对距离, 使用半定规划的方式求解 出其他目标点的三维坐标。

假设共有 $n$ 个点, 其中 $m$ 个待求点, $m_{a}$ 个针点。 建立如下的目标函数

$$
\sum_{(p, q) \in \mathcal{N}_{x}}\left(\hat{d}_{p q}^{2}-\left\|x_{p}-x_{q}\right\|^{2}\right)^{2}+\sum_{(p, r) \in \mathcal{N}_{a}}\left(\hat{d}_{p r}^{2}-\left\|x_{p}-a_{r}\right\|^{2}\right)^{2}
$$

式中, $\mathcal{N}_{x}$ 为待求点对组成的集合, 利用双目视觉 测量其相对距离, $\mathcal{N}_{a}$ 为待求点与针点点对组成的 集合, 利用双目视觉测量其相对距离, 针点的三维 坐标由激光测量得到。

点对间相对距离的测量值分别是 $\hat{d}_{p q}\left((p, q) \in \mathcal{N}_{x}\right), \hat{d}_{p r}\left((p, r) \in \mathcal{N}_{a}\right)$ 。 $a_{r}$ 表示由激光 测量得到的三维坐标, $x_{p} 、 a_{p}$ 分别表示待求点三维 坐标的计算值和真实值。

$$
\text { 令 } d_{p q}=\left\|x_{p}-x_{q}\right\|, d_{p r}=\left\|x_{p}-a_{r}\right\|, \text { 引入一个 }
$$

$3 \times m$ 维的由待求点三维坐标构成的矩阵变量 $\boldsymbol{X}=\left(x_{1}, x_{2}, \cdots, x_{m}\right) \in \mathbf{R}^{3 \times m}$ ，则可得到如下表达式

$$
\begin{aligned}
& \left\{\begin{array}{l}
d_{p q}^{2}=\sum_{i=1}^{3} X_{i p}^{2}-2 \sum_{i=1}^{3} X_{i p} X_{i q}+\sum_{i=1}^{3} X_{i q}^{2} \quad(p, q) \in \mathcal{N}_{x} \\
d_{p r}^{2}=\sum_{i=1}^{3} X_{i p}^{2}-2 \sum_{i=1}^{3} X_{i p} a_{i r}+\left\|a_{r}\right\|^{2} \quad(p, r) \in \mathcal{N}_{a}
\end{array}\right. \\
& \text { 令 } \xi_{p q}=\hat{d}_{p q}^{2}-d_{p q}^{2}, \quad \xi_{p r}=\hat{d}_{p r}^{2}-d_{p r}^{2} \text { 可将多项式优 }
\end{aligned}
$$
化问题转化为二次优化问题, 再用绝对值代替平方 项 ${ }^{[11]}$, 优化问题可表示为

$$
\begin{gathered}
\min \quad \sum_{(p, q) \in \mathcal{N}_{x}}\left(\xi_{p q}^{+}+\xi_{p q}^{-}\right)+\sum_{(p, r) \in \mathcal{N}_{o}}\left(\xi_{p r}^{+}+\xi_{p r}^{-}\right) \\
\text {s.t. } d_{p q}^{2}=\sum_{i=1}^{3} X_{i p}^{2}-2 \sum_{i=1}^{3} X_{i p} X_{i q}+\sum_{i=1}^{3} X_{i q}^{2} \quad(p, q) \in \mathcal{N}_{x} \\
d_{p r}^{2}=\sum_{i=1}^{3} X_{i p}^{2}-2 \sum_{i=1}^{3} X_{i p} a_{i r}+\left\|a_{r}\right\|^{2} \quad(p, r) \in \mathcal{N}_{a} \\
\xi_{p q}^{+} \geqslant 0 \quad \xi_{p q}^{-} \geqslant 0 \quad(p, q) \in \mathcal{N}_{x} \quad \xi_{p r}^{+} \geqslant 0 \quad \xi_{p r}^{-} \geqslant 0 \quad(p, r) \in \mathcal{N}_{a} \\
\xi_{p q}^{+}=\max \left\{0, \xi_{p q}\right\} \quad \xi_{p q}^{-}=\min \left\{0, \xi_{p q}\right\} \\
\xi_{p r}^{+}=\max \left\{0, \xi_{p r}\right\} \quad \xi_{p r}^{-}=\min \left\{0, \xi_{p r}\right\}
\end{gathered}
$$

\subsection{SDP 松弛模型}

令 $\mathcal{C}_{i}=i p: 1 \leqslant p \leqslant m(1 \leqslant i \leqslant 3),\left(X_{i p}, i p \in \mathcal{C}_{i}\right)$ 为 $X_{i p}\left(i p \in \mathcal{C}_{i}\right)$ 按照下标顺序构成的行矢量, 则可得到 以下表达式 ${ }^{[11]}$ 


$$
\begin{gathered}
\sum_{i p \in \mathcal{C}_{i}} \sum_{i q \in \mathcal{C}_{i}} \boldsymbol{E}\left(\mathcal{C}_{i}\right)_{i p i q} X_{i p} X_{i q}- \\
\left(X_{i p}, i p \in \mathcal{C}_{i}\right)^{\mathrm{T}}\left(X_{i p}, i p \in \mathcal{C}_{i}\right)=\boldsymbol{O}
\end{gathered}
$$

式中, $\boldsymbol{E}\left(\mathcal{C}_{i}\right)_{i p i q}$ 表示 $m \times m$ 维的对称矩阵, 其第 $(i p, i q)$ 及 $(i q, i p)$ 个元素是 1 , 其他元素为 0 。将该等式松驰 化可得

$$
\begin{gathered}
\sum_{i p \in \mathcal{C}_{i}} \sum_{i q \in \mathcal{C}_{i}} \boldsymbol{E}\left(\mathcal{C}_{i}\right)_{i p i q} X_{i p} X_{i q}- \\
\left(X_{i p}, i p \in \mathcal{C}_{i}\right)^{\mathrm{T}}\left(X_{i p}, i p \in \mathcal{C}_{i}\right) \succeq \boldsymbol{O} \\
\text { 令 } \\
Y_{p q}=\sum_{i=1}^{3} X_{i p} X_{i q} \quad \boldsymbol{Y}=\sum_{i=1}^{3}\left(\sum_{i p \in \mathcal{C}_{i}} \sum_{i q \in \mathcal{C}_{i}} \boldsymbol{E}\left(\mathcal{C}_{i}\right)_{i p i q} Y_{p q}\right)
\end{gathered}
$$

而

$$
\boldsymbol{X}=\sum_{i=1}^{3}\left(X_{i p}, i p \in \mathcal{C}_{i}\right)^{\mathrm{T}} \quad X_{i p} \quad i p \in \mathcal{C}_{i}
$$

可得 $\boldsymbol{Y}-\boldsymbol{X}^{\mathrm{T}} \boldsymbol{X} \succeq \boldsymbol{O}$, 根据 Schur 补定理可得

$$
\left(\begin{array}{cc}
\boldsymbol{I}_{t} & \boldsymbol{X} \\
\boldsymbol{X}^{\mathrm{T}} & \boldsymbol{Y}
\end{array}\right) \succeq \boldsymbol{O}
$$

则式(9)的全半定规划 ${ }^{[12]}$ 形式为

$$
\min \sum_{(p, q) \in \mathcal{N}_{x}}\left(\xi_{p q}^{+}+\xi_{p q}^{-}\right)+\sum_{(p, r) \in \mathcal{N}_{a}}\left(\xi_{p r}^{+}+\xi_{p r}^{-}\right)
$$

s.t. $\quad d_{p q}^{2}=\sum_{i=1}^{3} X_{i p}^{2}-2 \sum_{i=1}^{3} X_{i p} X_{i q}+\sum_{i=1}^{3} X_{i q}^{2} \quad(p, q) \in \mathcal{N}_{x}$

$$
d_{p r}^{2}=\sum_{i=1}^{3} X_{i p}^{2}-2 \sum_{i=1}^{3} X_{i p} a_{i r}+\left\|a_{r}\right\|^{2} \quad(p, r) \in \mathcal{N}_{a}
$$

$\xi_{p q}^{+} \geqslant 0 \quad \xi_{p q}^{-} \geqslant 0 \quad(p, q) \in \mathcal{N}_{x} \quad \xi_{p r}^{+} \geqslant 0 \quad \xi_{p r}^{-} \geqslant 0 \quad(p, r) \in \mathcal{N}$

$$
\left(\begin{array}{cc}
\boldsymbol{I}_{t} & \boldsymbol{X} \\
\boldsymbol{X}^{\mathrm{T}} & \boldsymbol{Y}
\end{array}\right) \succeq \boldsymbol{O}_{a}
$$

可将公式写成如下标准半定规划形式

$$
\begin{array}{ll}
\min & \boldsymbol{A}_{0} \cdot \boldsymbol{Z} \\
\text { s.t. } & \boldsymbol{A}_{t} \cdot \boldsymbol{Z}=b_{t} \quad t \in T \\
& \boldsymbol{Z} \succeq \boldsymbol{O}
\end{array}
$$

利用 SeDuMi 工具包 ${ }^{[12]}$ 可对半定规划问题进行 求解。

\section{3 仿真}

为了研究影响三维坐标求解精度的因素, 利用 Matlab 进行仿真, 假设

$$
\left\{\begin{array}{l}
\mathcal{N}_{x}=\left\{(p, q): 1 \leqslant p<q \leqslant m,\left\|a_{p}-a_{q}\right\| \leqslant \rho\right\} \\
\mathcal{N}_{a}=\left\{(p, r): 1 \leqslant p \leqslant m, m+1 \leqslant r \leqslant n,\left\|a_{p}-a_{r}\right\| \leqslant \rho\right\}
\end{array}\right.
$$

待求点三维坐标求解的均方误差为

$$
Y=\left(\frac{1}{m} \sum_{p=1}^{m}\left\|x_{p}-a_{p}\right\|^{2}\right)^{1 / 2}
$$

模拟隧道中的标志点, 隧道直径为 $8 \mathrm{~m}$, 生成 均匀分布的 30 个待求点, 并生成尽量分散的 15 个 点作为针点。得到如图 13 所示的点。其中, 空心点 表示待求点, 实心点表示针点。

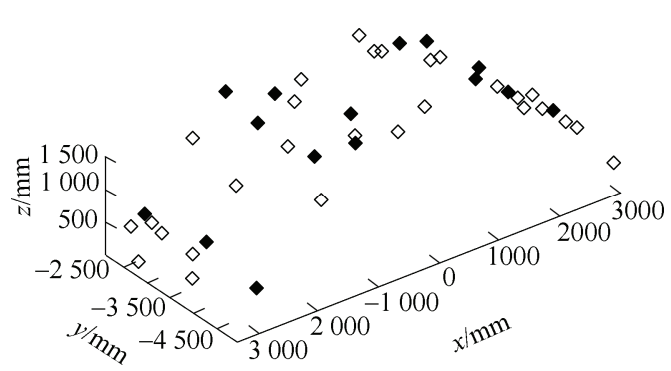

图 13 仿真的待求点和针点

假设双目视觉距离测量误差 $\sigma$ 为 $2 \mathrm{~mm}$, 如图 14 所示为均方误差 $r$ 与最大目标点相对距离 $\rho$ 的关 系, $r$ 随着 $\rho$ 的增大而减小, 当 $\rho$ 大于一定值时, $r$ 保持不变, 其原因是 $\mathcal{N}_{x}$ 和 $\mathcal{N}_{a}$ 已取得最大值, 因此 为了获得较高的精度, $\mathcal{N}_{x}$ 和 $\mathcal{N}_{a}$ 尽量取最大值。

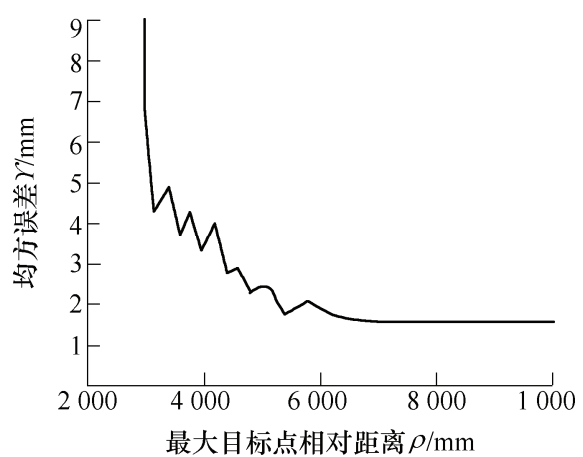

图 $14 r$ 与 $\rho$ 之间的关系

取 $\rho=8000 \mathrm{~mm}$, 随机的从 15 个针点中取出 5 至 15 个针点, 其误差箱线图如图 15 所示, 从图中 可以看出当针点数大于某一值时，不同针点数的误 差相差不大。图中选择 10 个针点数较为合适。

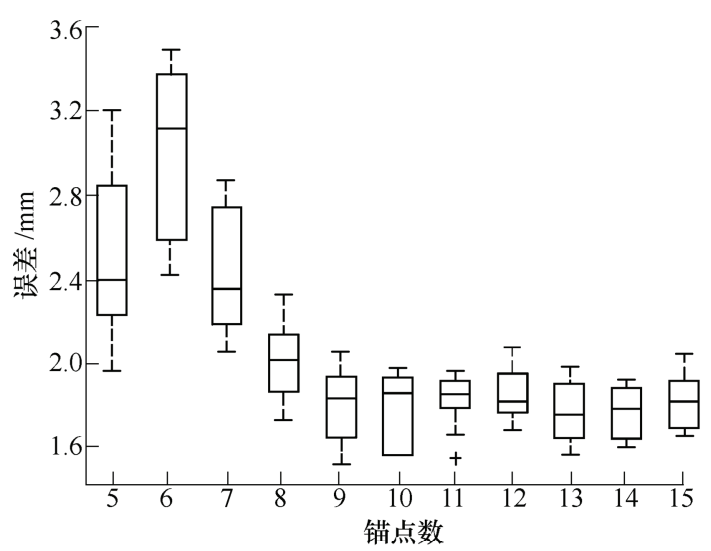

图 15 三维坐标求解误差箱线图 
当 $\rho=8000 \mathrm{~mm}$, 针点数选为 10 时, 改变双目 视觉测量的距离误差 $\sigma$, 可得到 $Y$ 与 $\sigma$ 的关系如图 16 所示。其中实线表示待求点的求解误差, 虚线表 示激光测量误差为 $1 \mathrm{~mm}$ 时系统的总体误差。从图 中可看出双目视觉的距离误差为 $0.5 \sim 1.5 \mathrm{~mm}$ 较为 合适。选取基线长度为 $1 \mathrm{~m}$, 两个型号为 MV-EM1400C 的工业相机、型号为 M1214-MP2 的 工业镜头完成双目测距。

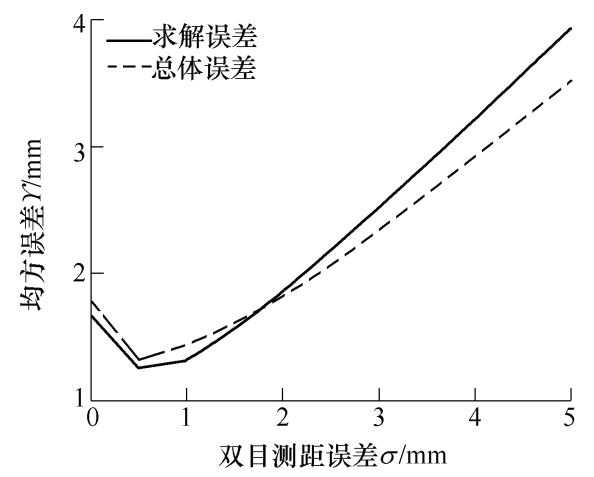

图 $16 r$ 与 $\sigma$ 之间的关系图 1

如图 17 所示, 求解误差 1 曲线和求解误差 2 曲线分别表示激光测量误差为 $1 \mathrm{~mm}$ 和激光测量无 误差时的待求点的坐标求解误差。从图中可以看出 当激光测量无误差时, 待求点的求解误差与双目视 觉测量的距离误差近似成正比关系, 原因在于梯度 下降法将计算误差消除, 使得求解误差只与距离误 差有关。激光测量误差会影响待求点的坐标求解误 差, 并使之增大。

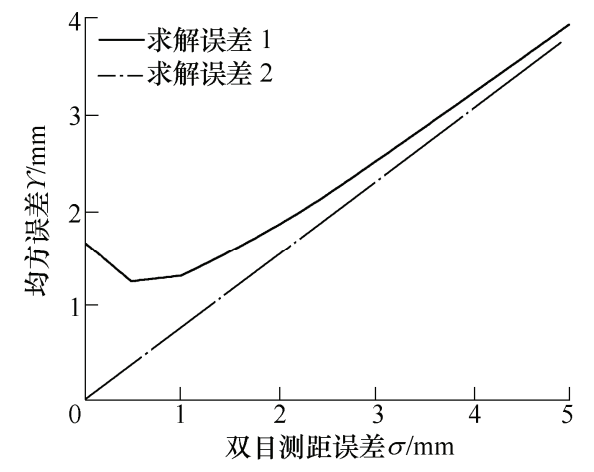

图 $17 r$ 与 $\sigma$ 之间的关系 2

根据实际情况, 假设激光测量每个点的时间为 $20 \mathrm{~s}$, 双目视觉测量所有点的相对距离所用时间为 $20 \mathrm{~s}$ 。当双目视觉的距离测量精度分别为 $0.5 \mathrm{~mm}$ 、

$0.8 \mathrm{~mm} 、 1 \mathrm{~mm}$ 时, 利用半定规划求解的精度损失 率与测量速度增益率的关系如图 18 所示。

从图中可看出, 虽然针点的数目的确会对精度 损失造成一定的影响, 速度增益率在一定的范围内 精度损失率相差不大, 当速度增益率大于某一值时
精度损失率将显著增加。

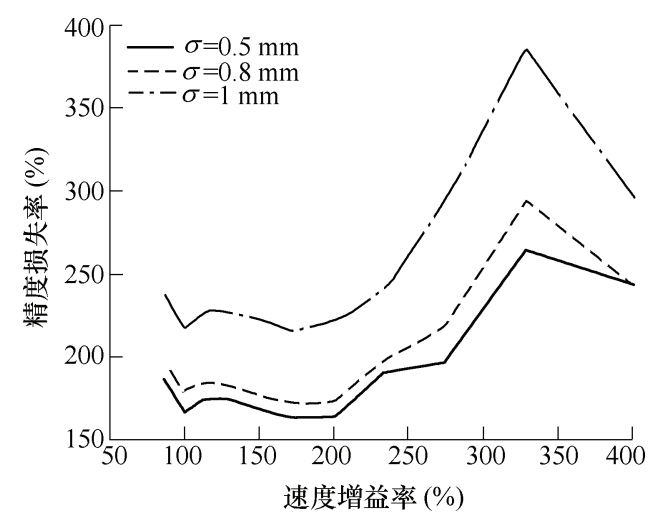

图 18 精度损失率与速度增益率之间的关系

待求点三维坐标求解值与真实值的对比如图 19 所示, 其中圆圈是待求点的实际坐标, 星点是 待求点的计算坐标, 菱形是针点。将其误差放大 200 倍后的效果如图 20 所示。从图中可以看出, 坚直方向的误差较大, 主要是由距离向量在坚直 方向上的分量较小, 且坚直方向上的点分布较为 集中导致的。

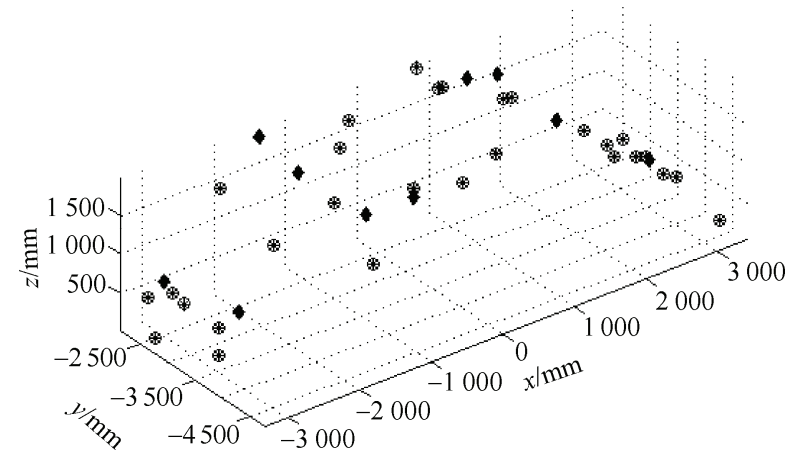

图 19 三维坐标的拟合效果图

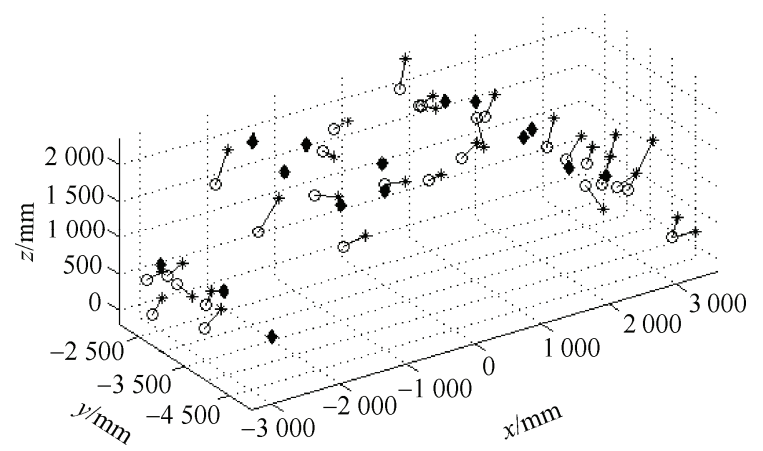

图 20 误差放大 200 倍后三维坐标的拟合效果图

\section{5 试验}

如图 21 所示搭建了试验平台, 并设计了如图 22 所示的试验板。将试验装置标定后, 利用试验装 置对试验板上的 6 个标志点进行测量, 并利用三坐 标测量仪测量 6 个标志点的中心坐标。 


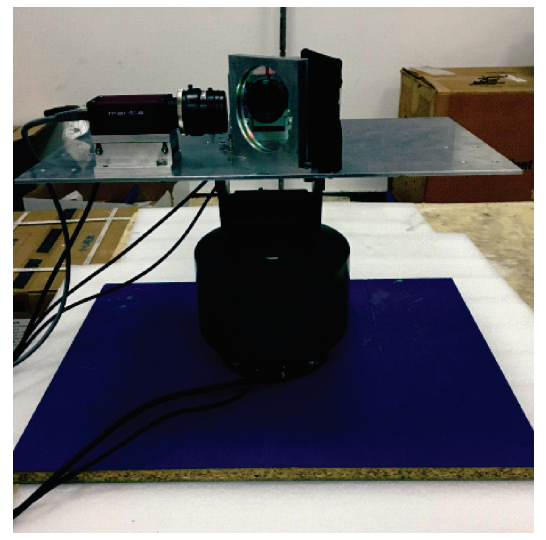

图 21 试验平台

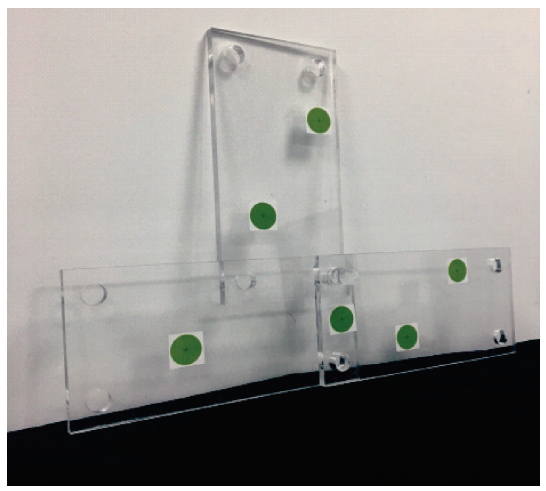

图 22 试验板

由于三坐标测量仪的精度在 $\mu \mathrm{m}$ 级, 可认为该 结果为真实值, 将试验装置的测量值利用最小二乘 法转化到三坐标测量仪的坐标系下, 并与三坐标测 量仪测得结果进行对比, 分别在三个不同位置进行 了测量, 其误差箱线图如图 23 所示。从图 23 中可 看出, 试验装置的测量误差小于 $1 \mathrm{~mm}$, 并且在已有 的硬件环境下测量一个点在 $20 \mathrm{~s}$ 以内。因此试验装 置具有较高的测量精度和效率。

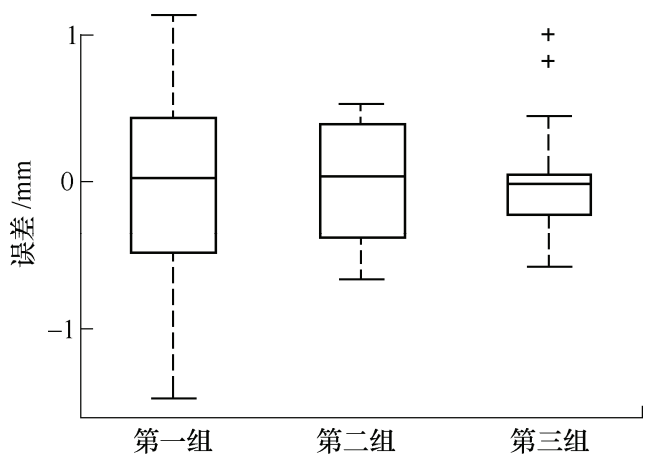

图 23 试验装置测量误差箱线图

\section{6 结论}

本文针对 TBM 掘进过程中实现自动、精确的 隧道轮廓监测的问题, 提出了监测方案并设计了测
量装置, 提出目标瞄准、测量和数据处理算法, 仿 真验证了结合双目视觉以提高测量效率的可行性。 系统能精确、快速地扫描空间的标志点, 其三维坐 标测量误差小于 $1 \mathrm{~mm}$ 。但是针对非圆标志点对测量 精度的影响以及现场试验等问题还有待进一步的分 析研究。

\section{参 考 文 献}

[1] 杜彦良, 杜立杰. 全断面岩石隧道掘进机系统原理与集 成设计[M]. 武汉: 华中科技大学出版社, 2011.

DU Yanliang, DU Lijie. Full face hard rock boring machine-system principles and integrated design[M]. Wuhan: Huazhong University of Science and Technology Press, 2011

[2] 苏华友, 任月宗, 薛继洪. 不良地质条件对 TBM 施工 的影响与探讨 [J]. 中国铇业, 2009, 24(2): 44-47.

SU Huayou, REN Yueming, XUE Jihong. Adverse geological conditions effect and analysis on tunnel boring machine construction[J]. China Tungsten Industry, 2009, 24(2): 44-47.

[3] BASSETT R H, KIMMANCE J P, RASMUSSEN C. An automated electrolevel deformation monitoring system for tunnels[J]. Proceedings of the ICE-Geotechnical Engineering, 1999, 137(3): 117-125.

[4] NARUSE H, KOMATSU K, FUJIHASHI K, et al. Telecommunications tunnel monitoring system based on distributed optical fiber strain measurement[J]. Proc. SPIE, 2005, 5855: 168-171.

[5] STURM P. Computer vision[M]. Boston: Springer, 2014.

[6] ZHANG Z. A flexible new technique for camera calibration[J]. IEEE Transactions on Pattern Analysis \& Machine Intelligence, 2000，22(11): 1330-1334.

[7] 刘媛媛, 赵冬娥. 对于斜入射滤光片的分析[J]. 电子世 界，2013(15): 28-29.

LIU Yuanyuan, ZHAO Donge. Analysis of oblique incidence filter[J]. Electronics World, 2013(15): 28-29.

[8] 张铮, 王艳平, 薛桂香. 数字图像处理与机器视觉 $[\mathrm{M}]$. 北京：人民邮电出版社，2010.

ZHANG Zhen, WANG Yanping, XUE Guixiang. Digital image processing and machine vision[M]. Beijing: Posts and Telecom Press, 2010.

[9] CANNY J. A computational approach to edge detection[J]. IEEE Transactions on Pattern Analysis and Machine Intelligence, 1986, 8(6): 679-98.

[10] CORKE P I, SPINDLER F, CHAUMETTE F. Combining Cartesian and polar coordinates in IBVS[C]// The 2009 IEEE/RSJ International Conference on Intelligent Robots and Systems. October 11-15, 2009, St. Louis, USA. New 
York: IEEE Press, 2009: 5962-5967.

[11] KIM S, KOJIMA M, WAKI H. Exploiting sparsity in SDP relaxation for sensor network localization[J]. SIAM Journal on Optimization, 2009， 20(1): 192-215.

[12] BISWAS P, YE Y. Semidefinite programming for ad hoc wireless sensor network localization[C]// International Symposium on Information Processing in Sensor Networks. April 26-27, 2004, Berkeley, California, USA. New York: ACM, 2004: 46-54.

[13] STURM J F. Using SeDuMi 1.02, a MATLAB toolbox for optimization over symmetric constraints[J]. Optimization Methods and Software, 1998, 11(1-4): 625-653.

作者简介: 李华, 女, 1992 年出生。主要研究方向为地下工程表面的安 全监测。

E-mail:897345481@qq.com

雷勇(通信作者), 男, 1955 年出生, 博士, 副教授, 博士研究生导师。 主要研究方向为故障诊断、机器人、机器人辅助介入治疗。

E-mail: ylei@zju.edu.cn

甘创, 男, 1992 年出生, 硕士研究生。主要研究方向为地下工程表面的 安全监测。

E-mail: chuangganzju@163.com

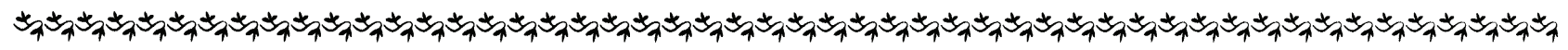

第 7 届上银优秀机械博士论文奖——铜奖

\section{超声能场在金属微/介观成形中的作用理论及实验研究}

作者: 姚喆赫

毕业学校: 浙江大学

指导教师：陈子辰 梅德庆

产品微型化和集成化的发展趋势, 极大地增加了对微小型金属零件和微/介观制造技术的需求。微/介观金属成形技术为 微小型金属零件的大批量、高效率的制造提供了解决方案。然而, 将传统金属成形技术缩小至微/介观尺度所带来的尺度效应 导致了金属成形性下降和工件表面质量下降等问题, 制约了微/介观成形技术的发展。利用超声振动在金属成形过程中所具备 的体积效应和表面效应, 在微/介观成形中施加超声振动有望解决上述问题。为此, 本文结合国家自然科学基金重点项目(项 目编号: 50930005)、国家自然科学基金面上项目(项目编号: 50775203)以及美国自然科学基金项目(项目编号: CMMI-0800353), 采用理论分析、数值模拟和试验研究相结合的方式, 开展了超声振动在金属微/介观成形中的作用机理及超声辅助金属微/介 观成形的制造工艺研究。

第一章阐述了本论文研究的背景与意义, 结合相关研究的国内外研究综述, 提出了本论文的主要研究内容; 第二章和第 三章研究了超声振动的体积效应, 其中, 第二章结合理论和试验研究探索了超声能场中的金属声软化效应; 第三章探索了超 声能场中的金属声残余硬化效应, 并构建了声塑性理论模型; 第四章和第五章研究了超声振动的表面效应, 其中, 第四章研 究了超声振动对金属成形工件表面微观形貌的影响机理; 第五章研究了超声振动对于微/介观镦锻成形中接触摩擦的影响机 理; 第二章至第五章研究了超声振动对于固态金属微/介观成形的影响规律，第六章则研究了超声振动对于半固态金属微/介 观成形的作用机理; 基于第二章至第六章的机理研究, 第七章研制了分别用于固态金属和半固态金属的超声辅助成形制造系 统，并开展了工艺试验研究；第八章对于全文的研究工作进行了总结与展望。

本文研究的创新之处在于以下几点。

(1) 在晶体塑性理论框架下, 研究了超声能场在热激活过程和位错演化过程中的作用机理, 进而构建了声塑性理论模型, 可准确描述超声辅助金属成形中的声软化和声残余硬化的作用机制。

(2) 系统研究了超声振动在金属微/介观成形中的体积效应和表面效应，并基于试验方法发现并识别了超声辅助金属微/ 介观成形中的金属声软化效应和声残余硬化效应的耦合作用机制。

(3) 基于声软化效应理论, 建立了超声振动对金属表面微观形貌以及接触摩擦因子的影响理论模型, 提出了超声处理后 的表面粗粘度预测方法以及镦锻成形试验中摩擦因子的估算方法。

(4) 针对半固态金属微/介观成形中存在的尺度效应及充型缺陷问题, 将超声振动引入半固态金属微/介观成形工艺中, 提 出了一种超声辅助半固态金属微/介观成形新工艺, 并研制了制造系统, 进行了工艺试验和应用研究。

研究结果表明将超声能场引入金属微/介观成形和半固态金属微/介观成形工艺中, 能够降低成形载荷, 改善特征结构成 形效果, 也能够改善工件表面质量, 降低界面摩擦阻力, 从而可在体积因素和表面因素两方面有效克服微/介观尺度效应引起 的不利影响。本文提出的超声辅助半固态金属微/介观成形技术为微细零件的工业化制造提供了新方法。研究成果对于深入探 索超声振动在金属材料中的作用机制以及超声辅助金属微/介观成形制造系统的设计开发等方面具有重要的理论意义, 并有望 应用于新能源、电子通讯、生物医学等领域的三维微细金属零件制造过程。 